\title{
An unusual presentation of scrub typhus in a child: a case report
}

\author{
Jimba Jatsho* ${ }^{*}$
}

\begin{abstract}
Background: Scrub Typhus (ST) is an acute, febrile zoonotic disease caused by the bacterium Orientia tsutsugamushi which is endemic to the Asia-Pacific region. Infected adults rarely present with sensorineural hearing loss and otalgia. Though few cases of pediatric cases are known to present with otalgia, no pediatric cases of sensorineural deafness complicating ST have been reported to date.

Case presentation: A 5-year-old, previously healthy girl presented with a one-week history of high-grade intermittent fever, Headache and right ear pain with a recent onset of reduced hearing. She had a fever up to $39^{\circ} \mathrm{C}$, cervical lymphadenopathy, bilateral pleural effusion with diffuse infiltrations, and mild hepatosplenomegaly with no evidence of rash. Her initial examination was normal except for mildly enlarged tonsils. Unilateral right ear hearing loss was noted using Weber's test. Evidence of progressive, mild anemia, and raised inflammatory markers was noted. Diagnosis of scrub typhus was confirmed by positive detection of Orentia tsutsugamushi IgM antibodies on rapid diagnostic test and the presence of chigger mite in the right external auditory canal on repeat examination. She responded dramatically to the empirical treatment of ceftriaxone and doxycycline. On follow-up, she did not have any residual hearing loss and her ear pain had resolved completely.
\end{abstract}

Conclusion: Acute onset hearing loss or severe otalgia with or without findings should be an important diagnostic clue for suspecting scrub typhus in patients who present with a history of fever especially in endemic areas.

Keywords: Scrub typhus, Rickettisia, Children, Sensorineural hearing loss, Otalgia, Bhutan

\section{Background}

Scrub typhus (ST) is a mite-borne rickettsial zoonosis caused by Orientia tsutsugamushi. The trombiculid mites (genus Leptotrombidium) act as the vector and the disease is transmitted to humans through the bite from the infected larval stages (chiggers) of the mites [1]. ST presents as a simple febrile illness with eschar and rash but can sometimes present with a myriad of atypical and unusual manifestations [2]. Neurologically, patients usually present with severe headaches but can occasionally manifest as meningitis and meningoencephalitis, acute disseminated encephalomyelitis, and cranial nerve palsies

*Correspondence: jimbajatsho@gmail.com

Department of Pediatrics, Phuentsholing Hospital, Ministry of Health, Phuentsholing, Bhutan
[3-5]. In rare instances, patients infected with Rickettsia rickettsii can present with sensorineural hearing loss and otalgia [6-8]. No pediatric cases of sensorineural deafness complicating ST have been reported to date.

We report a case of ST in a child presenting with severe otalgia and unilateral sensorineural hearing loss, an uncommon presentation of this neglected tropical disease.

\section{Case presentation}

A 5-year-old girl, a previously healthy child, presented with a one-week history of high-grade intermittent fever, headache, and right ear pain. She complained of paroxysmal right ear pain but without any history of ear discharge, tinnitus, dizziness, or seizures. She had a headache with nausea but no vomiting or loss of 
consciousness. This was associated with some abdominal distension and pain. Her bowel and urine output was normal, however, she had dysuria. The patient's mother denied any history of insect or tick bite even though the child usually plays in the fields during the day. Both parents are farmers.

On the 7th day after the onset of illness, she complained of reduced hearing in her right ear without tinnitus, which gradually progressed to unilateral hearing loss.

Physical examination was notable for fever of $39^{\circ} \mathrm{C}$ and cervical lymphadenopathy. There was no evidence of any rash or eschar. Examination of the throat revealed mildly enlarged tonsils without signs of inflammation. Initial otoscopic examination of the ears was unremarkable. Unilateral right ear sensorineural hearing loss was noted using Weber's tuning fork test since audiometric tests weren't available at our district hospital. She was mildly tachypneic but did not require oxygen. Bilateral pleural effusion was noted. Abdominal examination was notable for mild hepatosplenomegaly.

Laboratory tests revealed thrombocytopenia (platelets 76,000 cells $/ \mathrm{mm}^{3}$ ), mild anemia (hemoglobin $10.6 \mathrm{~g} / \mathrm{dL}$ ) and slightly raised neutrophils (70\%). Subsequently, over the next few days, her platelet counts gradually decreased to a nadir of 47,000 cells $/ \mathrm{mm}^{3}$. The C-reactive protein was $105 \mathrm{mg} / \mathrm{dL}$ with an increased erythrocyte sedimentation rate of $20 \mathrm{~mm} / \mathrm{hr}$. Urine revealed mild proteinuria. Chest X-ray showed bilateral pleural effusion with bilateral infiltrates (Fig. 1). An abdominal ultrasonogram confirmed mild hepatosplenomegaly. Rapid diagnostic tests (RDT) for leishmaniasis were negative but positive for dengue IgG. SD BIOLINE Tsutsugamushi RDT for scrub typhus was noted to be positive. SD BIOLINE Tsutsugamushi test is a rapid, qualitative, and differential test for the detection of Orientia Tsutsugamushi antibodies (IgG, IgM, IgA) in our setting [9].

Empiric treatment was started with intravenous ceftriaxone and oral doxycycline. On the 6th day of admission, an otoscopic examination was repeated because of persistent mild ear pain and worsening hearing impairment. This revealed a chigger mite in the external auditory canal which was promptly removed (Fig. 2). However, there was no evidence of otitis media or inner ear abnormalities. Her pain settled soon after the removal of the mite and her fever resolved 2 days after the initiation of antibiotics.

At one-week follow-up, she did not have any residual hearing loss and the otalgia had resolved completely.

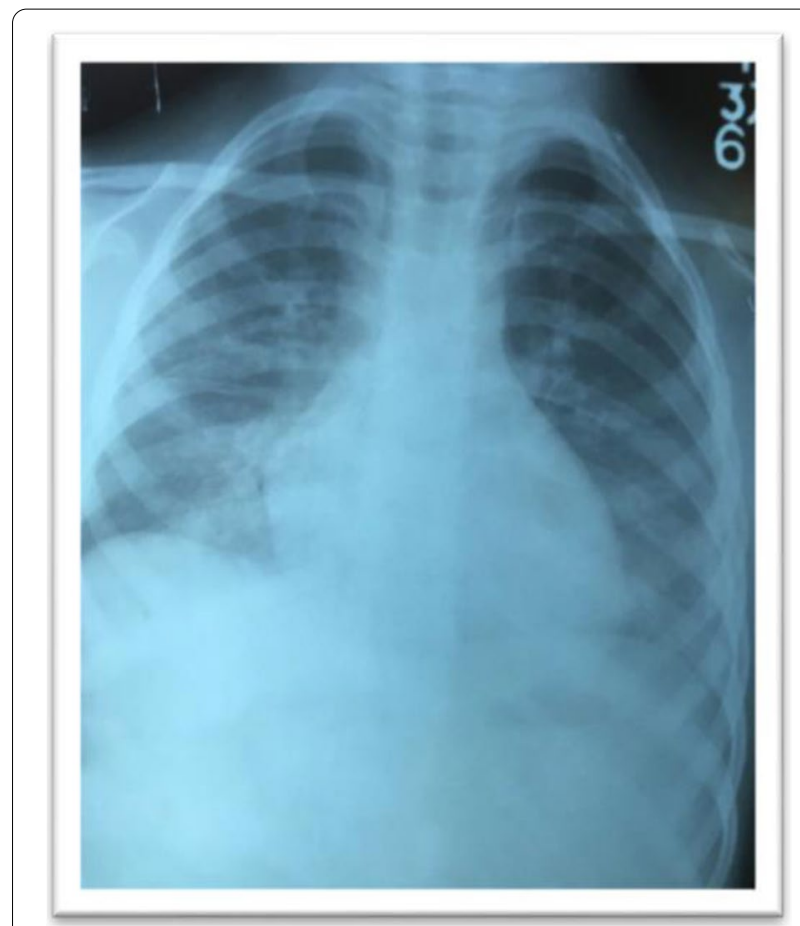

Fig. 1 Chest X-ray showing minimal pleural effusion with bilateral diffuse infiltrates

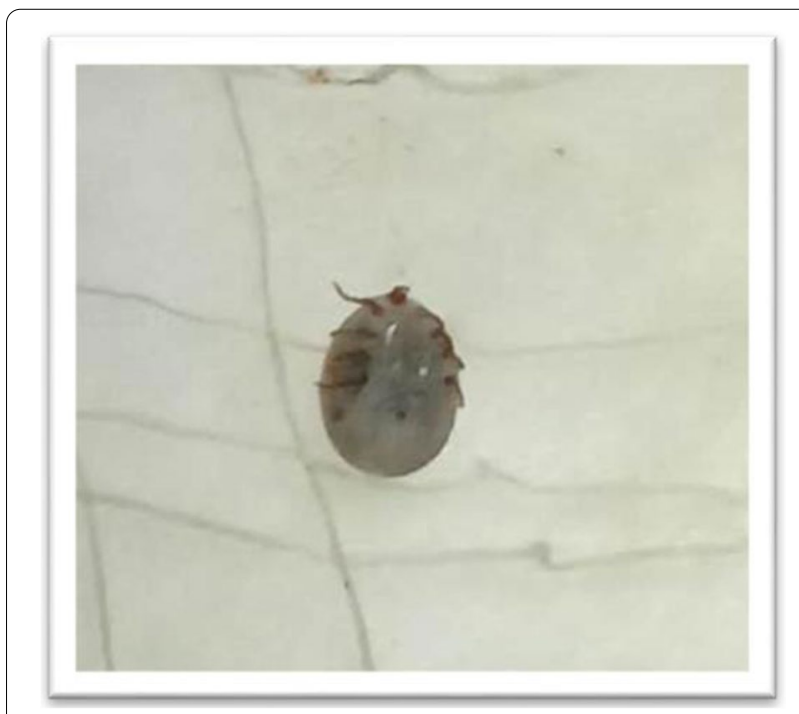

Fig. 2 Trombiculid chigger mite removed from right external auditory canal

\section{Discussion and conclusions}

Bhutan lies within the endemic area of scrub typhus historically referred to as the "tsutsugamushi triangle" [10]. ST cases have been reported since 2009 and became a 
notifiable disease of public health importance from 2010 [11] A recent serosurvey showed a high seroprevalence of scrub typhus and rickettsia in Bhutan [12]. In Bhutan, cases were likely underreported given the nonspecific clinical presentation, misdiagnosis by clinicians, and a lack of awareness of the disease [11].

Clinically, ST has a diverse clinical manifestation ranging from nonspecific febrile illness, rash, eschar, headache, myalgia, cough, generalized lymphadenopathy, nausea, vomiting, and abdominal pain to severe multiorgan dysfunction [13]. Fever and headache were the most common presenting symptoms [10]. The presence of an eschar, which is a typical painless necrotic skin lesion that may develop at the site of the bite, is pathognomonic. However, these are not present in several rickettsial diseases especially in children [14]. In Bhutan, common symptoms were nonspecific, and an eschar was noted by clinicians in only $7.4 \%$ of cases [12].

Central nervous system (CNS) manifestations are an uncommon and atypical feature of ST. Published case reports on CNS manifestations of ST in the pediatric population are limited. Among these, meningitis and meningoencephalitis are commonly reported with a subset of severe forms of patients presenting with altered sensorium and seizures [2]. In 2014, outbreaks of ST in a remote school in Bhutan lead to 2 deaths from meningoencephalitis [15].

Otalgia with sensorineural deafness is an unusual manifestation of ST. To date, no pediatric cases of sensorineural deafness complicating ST have been reported. Most of these cases described in the literature are in adult patients $[6,8,16]$. Premaratne et al. noted that hearing loss was a presenting symptom in $19 \%$ of patients with ST and affected up to one-third of the patients [8].

Our patient presented with nonspecific fever, headache, and associated sensorineural hearing loss, and severe otalgia. A sensory neural type of hearing loss has been reported in patients with rickettsial infections [7, $8,17]$. The actual mechanism of hearing loss in rickettsial diseases like ST is unclear. However, two possible pathological mechanisms have been postulated. Firstly, vasculitis-induced cochlear damage in the acute stage and secondly, an immune-mediated vasculitis in the vasa vasorum of the cochlear nerve $[18,19]$ have been proposed. In children, rickettsial infections are noted as a cause of secondary vasculitis [20].

In previous case reports in adults, otalgia usually appeared within the first week, followed by hearing loss and tinnitus in the second week after the onset of ST [6, 8]. Comparatively, in our patient, hearing loss appeared earlier within the first week of illness after the onset of otalgia. However, the reason for this early hearing loss in children is not known. Removal of the trigger of the inciting condition leads to remission of the vasculitis [20]. This corroborates the self-limiting course of hearing loss as in our patient.

Timely diagnosis and effective antibiotic treatment are effective in achieving good clinical outcomes [6]. However, in resource-limited settings like ours, mostly affordable RDTs allow rapid point-of-care testing and diagnosis. Serological tests like indirect immunofluorescence assays (IFAs) though sensitive are limited by the lack of standardization, variable cutoff titers for endemic regions, the requirement for paired sera, and high cost [21]. Other diagnostic methods include biopsy of eschar, culture, and polymerase chain reaction (PCR) including nucleic acid amplification (NAA). Early detection and diagnostic accuracy can be improved by combining NAA and IgM RDTs or Enzyme-Linked Immunosorbent Assays (ELISA) [22].

Clinicians working in endemic areas must consider ST and other rickettsial diseases in patients presenting with fever and sensorineural hearing loss or severe otalgia with or without otoscopic abnormalities.

\section{Abbreviations}

ST: Scrub Typhus; CNS: Central nervous system; RDT: Rapid Diagnostic Test; NAA: Nucleic acid amplification; PCR: Polymerase chain reaction; IFA: Immunofluorescence assays; ELISA: Enzyme-Linked Immunosorbent Assays.

\section{Acknowledgement}

The author would like to thank the administration and staff of the pediatric department at Phuentsholing hospital for their support.

\section{Author's contributions}

JJ was responsible for the conception, acquisition of details and design of this case report. JJ approves the submitted version and agrees to be personally accountable for the contributions and any issues related to the accuracy or integrity of any part of this work.

Funding

No funding was obtained for this case report.

Availability of data and materials

Data on patient and case details are available from the author on reasonable request.

\section{Declarations}

\section{Ethics approval and consent to participate}

Initially informed verbal consent was granted by the patient's parents for inclusion in this case report. However as per requirement, signed informed consent was further obtained for participation and reporting of the case as approved by the Phuentsholing General Hospital ethics administration, Chukha, Bhutan.

\section{Consent for publication}

The patient's parents gave their written consent for their child's clinical details and related investigation reports (Chest X-ray) to be published in this study.

\section{Competing interests}

The author declares no competing interests. 
Received: 7 April 2021 Accepted: 26 January 2022

Published online: 03 February 2022

\section{References}

1. Pham XD, Otsuka Y, Suzuki H, et al. Detection of Orientia tsutsugamushi (Rickettsiales: rickettsiaceae) in unengorged chiggers (Acari:Trombiculidae) from Oita prefecture, Japan, by nested polymerase chain reaction. $J$ Med Entomol. 2001;38:308-11.

2. Rajapaksea S, Weeratungab P, Sivayoganathana S, Fernandoc SD. Clinical manifestations of scrub typhus. Trans R Soc Trop Med Hyg 2017;111:43-54

3. Misra UK, Kalita J, Mani VE. Neurological manifestations of scrub typhus. J Neurol Neurosurg Psychiatry. 2015;86:761-6. https://doi.org/10.1136/ jnnp-2014-308722.

4. Chen PH, Hung KH, Cheng SJ, et al. Scrub typhus-associated acute disseminated encephalomyelitis. Acta Neurol Taiwanica. 2006;15:251-4.

5. Bhardwaj B, Panda P, Revannasiddaiah S, et al. Abducens nerve palsy in a patient with scrub typhus: a case report. Trop Biomed. 2013;30:706-9.

6. Kang Jl, Kim DM, Lee J. Acute sensorineural hearing loss and severe otalgia due to scrub typhus. BMC Infect Dis. 2009;9:173. Published 2009 Oct 22. https://doi.org/10.1186/1471-2334-9-173.

7. Tsiachris D, Deutsch M, Vassilopoulos D, Zafiropoulou R, Archimandritis AJ. Sensorineural hearing loss complicating severe rickettsial diseases: report of two cases. J Inf Secur. 2008;56(1):74-6. https://doi.org/10.1016/j. jinf.2007.10.002.

8. Premaratna R, Chandrasena TG, Dassayake AS, Loftis AD, Dasch GA, de Silva HJ. Acute hearing loss due to scrub typhus: a forgotten complication of a reemerging disease. Clin Infect Dis. 2006;42(1):e6-8. https://doi. org/10.1086/498747.

9. Silpasakorn S, Waywa D, Hoontrakul S, Suttinont C, Losuwanaluk K, Suputtamongkol Y. Performance of SD Bioline Tsutsugamushi assays for the diagnosis of scrub typhus in Thailand. J Med Assoc Thail. 2012;95(Suppl 2):S18-22 PMID: 22574525.

10. Xu G, Walker DH, Jupiter D, Melby PC, Arcari CM. A review of the global epidemiology of scrub typhus. PLoS Negl Trop Dis. 2017;11(11):e0006062. https://doi.org/10.1371/journal.pntd.0006062.

11. Dorji K, Phuentshok Y, Zangpo T, et al. Clinical and epidemiological patterns of scrub typhus, an emerging disease in Bhutan. Trop Med Infect Dis. 2019;4(2):56. Published 2019 Mar 29. https://doi.org/10.3390/tropi calmed4020056.

12. Tshokey T, Stenos J, Durrheim DN, Eastwood K, Nguyen C, et al. Seroprevalence of rickettsial infections and Q fever in Bhutan. PLoS Negl Trop Dis. 2017;11(11):e0006107. https://doi.org/10.1371/journal.pntd.0006107.

13. Sood AK, Chauhan L, Gupta H. CNS manifestations in Orientia tsutsugamushi disease (scrub typhus) in North India. Indian J Pediatr. 2016;83(7):634-9. https://doi.org/10.1007/s12098-015-2001-2.

14. Parola P, Paddock CD, Socolovschi C, et al. Update on tick-borne rickettsioses around the world: a geographic approach. Clin Microbiol Rev. 2013;26:657-702.

15. Tshokey T, Graves S, Tshering D, et al. Scrub Typhus Outbreak in a Remote Primary School, Bhutan,2014. Emerg Infect Dis. 2017;23(8):1412-4. https://doi.org/10.3201/eid2308.162021

16. Mahajan SK, Bakshi D. Acute reversible hearing loss in scrub typhus. J Assoc Physicians India. 2007;55:512-4.

17. Dolan S, Everett ED, Renner L. Hearing loss in Rocky Mountain spotted fever. Ann Intern Med. 1986;104:285.

18. Sexton DJ. Acute hearing loss and rickettsial diseases. Clin Infect Dis. 2006;42:1506

19. Walker DH, Parks FM, Betz TG, Taylor JP, Muehlberger JW. Histopathology and immunohistologic demonstration of the distribution of Rickettsia typhi in fatal murine typhus. Am J Clin Pathol. 1989;91:720-4.

20. Dedeoglu F, Sundel RP. Vasculitis in children. Rheum Dis Clin N Am. 2007;33(3):555-83. https://doi.org/10.1016/j.rdc.2007.07.007.

21. Blacksell SD, Bryant NJ, Paris DH, Doust JA, Sakoda Y, Day NP. Scrub typhus serologic testing with the indirect immunofluorescence method as a diagnostic gold standard: a lack of consensus leads to a lot of confusion. Clin Infect Dis. 2007:44(3):391-401.

22. Paris DH, Dumler JS. State of the art of diagnosis of rickettsial diseases: the use of blood specimens for diagnosis of scrub typhus, spotted fever group rickettsiosis, and murine typhus. Curr Opin Infect Dis. 2016;29(5):433

\section{Publisher's Note}

Springer Nature remains neutral with regard to jurisdictional claims in published maps and institutional affiliations.
Ready to submit your research? Choose BMC and benefit from:

- fast, convenient online submission

- thorough peer review by experienced researchers in your field

- rapid publication on acceptance

- support for research data, including large and complex data types

- gold Open Access which fosters wider collaboration and increased citations

- maximum visibility for your research: over $100 \mathrm{M}$ website views per year

At BMC, research is always in progress.

Learn more biomedcentral.com/submissions 